\title{
Multidrug-resistant Gram-negative Bacterial Infections in Critically Ill
}

\author{
Manohar Gandhi ${ }^{1}$, Rakshay Shetty ${ }^{2}$
}

\begin{abstract}
Antimicrobial resistance (AMR) in gram-negative bacteria (GNB) is persisting to be a significant cause of severe infections across the world, with increasing morbidity and mortality rates. Extended spectrum beta lactamase (ESBL) rates are alarmingly increasing in Escherichia and Klebsiella species, which is about $70 \%$ and there has been an increase in the resistance to carbapenems over the past few years in India. Current scenario of rapidly growing multidrug resistant (MDR) organisms in our Indian intensive care units is posing difficulties with regard to detecting these infections and starting appropriate empirical antibiotics. A clear understanding of the epidemiological, microbiological, and pharmacological aspects of these MDR gram-negative organisms is very important. This article tries to brief the risk factors for MDR GNB infections, spectrum of MDR GNB infections, mechanisms of resistance, and beta-lactamase enzyme classification and outlines the clinically important types and the treatment considerations for these MDR gram-negative organisms.
\end{abstract}

Keywords: Beta lactamases, Extended spectrum beta lactamase, Multidrug resistant gram-negative bacteria.

Pediatric Infectious Disease (2019): 10.5005/jp-journals-10081-1214

\section{INTRODUCTION}

Enterococcus faecium, Staphylococcus aureus, Klebsiella pneumoniae, Acinetobacter species, Pseudomonas aeruginosa, and Enterobacter species (ESKAPE) are the common multidrug resistant (MDR) organisms; of these organisms, the most important emerging threats are gram-negative bacilli. ${ }^{1}$ Antimicrobial resistance (AMR) in gram-negative bacteria (GNB) is persisting to be a significant cause of severe infections across the world, with increasing morbidity and mortality rates. ${ }^{1}$

It is mainly due to the presence of beta lactamases, which accounts for the widespread infections. Beta lactamases are of diverse types, of which extended spectrum beta lactamases (ESBLs) and carbapenemases are most important. ${ }^{2}$ Carbapenem resistance is increasing in the last few years in India. ${ }^{3}$ Extended spectrum beta lactamase rates are alarmingly increasing in E. coli and Klebsiella spp., which accounts for about $70 \%$. Three hundred and seventy percentage ICU patients surveyed in India showed resistance to multiple antibiotics, prevalence of metallobeta lactamase (MBL)-producing organisms ranges from $7 \%$ to $65 \%$ in India. ${ }^{4}$ Hospital-acquired Klebsiella infections which are resistant to carbapenem increased from $2 \%$ in 2001 to $10 \%$ in $2011 .{ }^{1,5}$ This article tries to brief the risk factors associated with MDR GNB, types of infections caused by MDR GNB, various mechanisms of resistance, and beta-lactamase classification and outlines the clinically important types and the treatment considerations for these MDR gram-negative organisms in critically ill.

\section{EPIDEMIOLOGY}

Study for monitoring antimicrobial resistance trends (SMART) which was done during 2004 shows that ESBL-producing bacteria were highest in Latin America, the Middle East, Africa, and Asia. ${ }^{6}$ The SMART has showed that prevalence of cephalosporin resistance mediated by ESBL) production in Escherichia coli and Klebsiella pneumoniae is $40.8 \%$ and $21.5 \%$, respectively, in the Asia-Pacific region. ${ }^{7}$

Community-acquired urinary tract infections (UTIs) caused by ESBL-producing E. coli which are resistant to cephalosporins and fluroquinolones are increasing in the US, Europe, and Asia. ${ }^{8-10}$
1,2Department of Pediatric Intensive Care and Emergency Medicine, Rainbow Children's Hospital, Bengaluru, Karnataka, India

Corresponding Author: Rakshay Shetty, Department of Pediatric Intensive Care and Emergency Medicine, Rainbow Children's Hospital, Bengaluru, Karnataka, India, Phone: +91 9535475354, e-mail: rakshayshetty@gmail.com

How to cite this article: Gandhi M, Shetty R. Multidrug-resistant Gram-negative Bacterial Infections in Critically III. Pediatr Inf Dis 2019;1(2):62-67.

Source of support: Nil

Conflict of interest: None

In 2007, the prevalence of community-acquired ESBL-producing E. coli infections is as common as hospital-acquired ones and $79 \%$ of the E. coli isolates tested were ESBL producers (Table 1). ${ }^{11}$

Why do we have so much of MDR organisms?

- Decades of overuse and misuse of antibiotics.

- Excess use of antibacterial products by the public over the counter and animal industry.

- Inappropriate antibiotic therapy.

- Overzealous antibiotic prescription by clinicians.

- Colonization pressure by resistant pathogens.

Common MDR GNB are the following: ${ }^{3}$

- ESBL-producing Enterobacteriaceae-E. coli, Klebsiella spp.

- Carbapenem-resistant enterobacteriaceae (CRE)-Klebsiella, Enterobacter, etc.

- Nonfermenting gram-negatives-Pseudomonas, Acinetobacter, and Stenotrophomonas.

\section{Risk Factors for MDR GNB Infections}

- Community-acquired infections: diabetes mellitus, previous hospitalization, urine catheterization, previous beta-lactam antibiotic usage. ${ }^{13}$ 
Table 1: Differences in resistance patterns in gram-negative organisms between Indian and western $\mathrm{ICU}^{12}$

\begin{tabular}{lll}
\hline Parameters & Western world & India \\
\hline Common isolates & Gram +ve & Gram -ve \\
Prevalence of ESBL GNB & Less & High \\
ICU type & Closed type & Open type \\
Generic medicines & Few & Many \\
Antibiotic policy & Strict & Not strict \\
\hline
\end{tabular}

Table 2: Mechanism of resistance by GNB and targets

\begin{tabular}{ll}
\hline Mechanism of resistance & Antibiotics \\
\hline Loss of porins & Carbapenems (imepenem) \\
Beta lactamases & Betalactams \\
Efflux pumps & $\begin{array}{l}\text { B-lactams, quinolones, } \\
\text { aminoglycosides, tigecycline, } \\
\text { and chloramphenicol }\end{array}$ \\
Mutations in the targets & $\begin{array}{l}\text { Quinolones (DNA gyrase and } \\
\text { topoisomerase) }\end{array}$ \\
Ribosomal mutations & Tetracyclines, aminoglycosides \\
Bypassing targets & Trimethoprim, sulfonamides \\
Antibiotic modifying enzymes & Aminoglycosides, ciprofloxacin \\
\hline
\end{tabular}

- Hospital-acquired infections: invasive procedures, prolonged hospitalization, and previous use of cephalosporins and fluoroquinolones. ${ }^{13}$

- Risk factors for carbapenemase-producing Klebsiella pneumoniae infections are the following:

Severe underlying disease, diabetes mellitus, ICU admission, mechanical ventilation, and exposure to betalactams, and fluoroquinolones. ${ }^{14,15}$

\section{Definitions of MDR, Extensively Drug Resistance, and Pan-drug Resistance ${ }^{16}$}

- MDR: The isolates are not susceptible to at least one antibiotic in three or more antimicrobial categories.

- Extensively drug resistance (XDR): The isolates are not susceptible to at least one antibiotic in all but two or fewer antimicrobial categories (i.e., they are susceptible to only one or two categories).

- Pan-drug resistance (PDR): The isolates are not susceptible to all antibiotics in all antimicrobial categories (i.e., no agents tested as susceptible for that organism).

\section{Mechanisms for AMR in MDR GNB}

GNB can adopt various mechanisms of resistance to a single class or to multiple classes of antibiotics, as depicted in Table 2.

Beta-lactamase production is the most important mechanism of resistance to betalactams adopted by GNB, and beta-lactamase enzymes break the amide bond of the betalactam ring and inactivates the betalactams. ${ }^{17}$

\section{Ambler Classification of ESBLs ${ }^{18,19}$}

Beta lactamases are classified based on their molecular structure and aminoacid pattern.

There are four classes: Classes A, C, and D have serine at their active site, and class $B$ beta lactamases are also called MBLs as they have zinc at their active site. ${ }^{17}$

\section{Class $A$}

These ESBLs are plasmid mediated, and they inhibit all penicillins and cephalosporins (except cephamycins) and aztreonam; b-lactamase inhibitors can inhibit these enzymes. There are mainly three groups in class A: TEM, SHV and CTX-M, and TEM and SHV are active against ceftazidime than cefotaxime and were predominant during the $1980 \mathrm{~s}$ and 1990s; and CTX-M are more active against cefotaxime, and they are now the most frequent ESBLs worldwide since 1990s. ${ }^{20}$

In India, CTX-M-mediated ESBLs are more common, followed by TEM and SHV. ${ }^{3}$

Klebsiella pneumoniae carbapenemases (KPCs) also belong to class A enzymes, and they can hydrolyze the carbapenems. ${ }^{21}$

\section{Class $B$}

They are also called MLBs, and they are active against penicillins, cephalosporins, and carbapenems. Monobactams (aztreonam) are not affected.

Important types of MLBs are IMP, VIM, and NDM. ${ }^{21}$

In India, carbapenem-resistant E. coli and Klebsiella spp. are mainly due to the presence of MBLs NDM- $1 .^{3}$

\section{Class C}

AmpC enzymes are the most important ESBLs in this class.

These enzymes are constitutively produced by several enterobacteria like Enterobacter species, Citrobacter freundii, Serratia marcescens, Morganella, Proteus, and Providentia. Class $C$ betalactamase genes are also present in transferable plasmids. ${ }^{22}$ They inhibit penicillins and cephalosporins except cefepime and are not inhibited by $\beta$-lactamase inhibitors. The AmpC enzyme-producing gene is inducible, and normally it is suppressed and not detected in vitro, but in vivo it can cause resistance. ${ }^{22,23}$

Classes B and C ESBLs have a broad spectrum of activity and they were always encoded by chromosomal genes, and hence, they are confined to a particular bacterial species. ${ }^{17}$

\section{Class D}

Oxacillinases are the most important enzymes in this class, and they hydrolyze oxacillin efficiently, hence the name. They inhibit aztreonam and carbapenems. The clinically important oxacillinases are OXA-48 and OXA-181. OXA-48 has spread widely among various enterobacteria, and it is very active against imipenem. OXA-48 is usually produced along with an ESBL. ${ }^{24}$

Summary of $\beta$-lactamases is given in Table 3 .

\section{Carbapenem Resistance}

Carbapenemase enzyme production is the important resistance mechanism against carbapenems.

Carbapenemases are ESBLs which can inhibit broad-spectrum betalactams antibiotics, like penicillins, cephalosporins, and carbapenems. $^{26}$

There are specific ESBLs in all the classes which has the ability to hydrolyze carbapenems.

In class A, KPCs are clinically very important. They inhibit all betalactams, and as they are plasmid-based, they are easily transferred to other gram-negative species, such as Escherichia coli, Enterobacter, Pseudomonas, and Salmonella. ${ }^{26}$

In class B, clinically important enzymes are the IMP, VIM, SPM, and NDM. ${ }^{17}$

The New Delhi MBL (NDM-1) is very important in Indian perspective.

It was first isolated from a Swedish patient hospitalized with K. pneumoniae infection in India in 2008 , since then they have been 
Table 3: Classwise important beta lactamases and their spectrum ${ }^{25}$

\begin{tabular}{|c|c|c|c|}
\hline Class & Enzymes & Spectrum & Epidemiology \\
\hline \multirow[t]{2}{*}{ Class A } & ESBLs (TEM, SHV, CTX-M) & $\begin{array}{l}\text { Penicillins, cephalosporins except } \\
\text { cefamycins, aztreonam, inhibited by } \\
\text { beta-lactamase inhibitors }\end{array}$ & $\begin{array}{l}\text { Worldwide, community acquired and nosocomial } \\
\text { infections }\end{array}$ \\
\hline & KPC & $\begin{array}{l}\text { Penicillins, cephalosporins, aztreonam, and } \\
\text { carbapenems. }\end{array}$ & $\begin{array}{l}\text { Predominant in E. coli, Salmonella, Enterobacter, } \\
\text { and Klebsiella, KPC cause hospital outbreaks }\end{array}$ \\
\hline \multirow[t]{2}{*}{ Class B } & $\begin{array}{l}\text { Metallobeta lactamases } \\
\text { (NDM, VIM, IMP) }\end{array}$ & $\begin{array}{l}\text { Penicillins, cephalosporins, carbapenems. } \\
\text { But aztreonam is susceptible. }\end{array}$ & $\begin{array}{l}\text { Worldwide spread. Nosocomial outbreaks and } \\
\text { endemic situations }\end{array}$ \\
\hline & & Not inhibited by beta-lactamase inhibitor & \\
\hline \multirow[t]{2}{*}{ Class C } & Amp C (CMY, DHA, FOX) & $\begin{array}{l}\text { Penicillins, cephalosporins (except } \\
\text { cefepime), and aztreonam }\end{array}$ & $\begin{array}{l}\text { Worldwide spread, community and nosocomial } \\
\text { infections }\end{array}$ \\
\hline & & Not inhibited by beta-lactamase inhibitor & \\
\hline \multirow[t]{2}{*}{ Class D } & OXA (OXA-48, OXA-181) & Penicillins, carbapenems, and aztreonam & Nosocomial outbreaks. \\
\hline & & Not inhibited by beta-lactamase inhibitor & \\
\hline
\end{tabular}

reported in the US and UK primarily associated with travel history to India or Pakistan. ${ }^{27,28}$ They inhibit all betalactams except aztreonam.

They reside on mobile gene cassettes inserted into integrons which harbor additional antibiotic resistance genes leading to MDR. These genes are transferred to other species via transposons and plasmids. ${ }^{23,29}$

In class D, clinically important enzymes are OXA family, ${ }^{30}$ and they are found primarily in P. aeruginosa and Acinetobacter species. ${ }^{26}$

VIM (37\%), NDM (17\%) in Pseudomonas aeruginosa and OXA-23 (98\%), and NDM (22\%) in Acinetobacter baumannii are the most prevalent carbapenemases noted in India. ${ }^{31}$

Other resistance mechanisms against carbapenems are impermeability and efflux, and these are common in Pseudomonas species. ${ }^{35}$

\section{Fluoroquinolone Resistance}

They acquire fluroquinolone resistance by altering the drug targets (DNA gyrase and/or topoisomerase IV) and also by efflux pumps especially in E. coli ${ }^{1}$ and Pseudomonas aeruginosa. ${ }^{32}$

\section{Aminoglycoside Resistance}

They acquire aminoglycoside resistance by producing inactivating enzymes which can cause phosphorylation, adenylylation, or acetylation.

Alternatively, methylation of the 16S rRNA drug target causes resistance to the entire aminoglycoside class, including novel aminoglycosides. ${ }^{33}$ These methylases are plasmid-mediated and are spread among GNB along with carbapenemases leading to MDR. ${ }^{34}$

\section{Multidrug Resistance}

GNB can activate several mechanisms at a time, like ESBL production, loss of porin channels, efflux pumps when they are exposed to an antibiotic leading to MDR. Transfer of plasmids with ESBL genes and other inactivating enzyme genes leads to the development of MDR strains. Pseudomonas aeruginosa and Acinetobacter baumannii most likely to become MDR in this fashion. ${ }^{35}$

\section{Common Infections Caused by GNB}

Blood stream infections (BSIs), ventilator-associated pneumonias (VAP), intra-abdominal infections (IAIs), skin and soft tissue infections, and urinary tract infections (UTIs) are the most commonly encountered infections. ${ }^{2}$

The epic 2 study showed that lungs are the most common site (64\%), followed by abdominal infection (19\%) and BSI (15\%). ${ }^{36}$

\section{When should We Suspect ESBL and Carbapenemase- producing GNB?}

Based on antibiogram, if the isolate is resistant to third and fourth generation cephalosporins but sensitive to beta-lactamase inhibitors (tazobactam), ESBL production should be suspected. If the isolate is resistant to carbapenems also, carbapenemase production has to be suspected. ${ }^{26}$

Most Klebsiella and E. coli without carbapenemases have minimal inhibitory concentrations (MICs) to imepenem and meropenem that are $<0.5 \mu \mathrm{g} / \mathrm{mL}$. Overt resistance of Klebsiella and $E$. coli to any of the carbapenems should arise suspicion of carbapenemase producers.

\section{Treatment of MDR GNB}

Early suspicion of MDR GNB by risk factors and timely initiation of appropriate antibiotic is very important step in treating these infections, and it decreases the mortality and improves the survival rate. ${ }^{37,38}$ Starting with broad-spectrum antibiotics and once the susceptibility report is available the antibiotics has to be optimized to more appropriate and narrow spectrum. ${ }^{38}$

\section{Empirical Therapy}

Local institutionwise antibiograms have to be prepared based on the local prevalence of MDR organisms, ${ }^{39}$ and based on susceptibility patterns, broad-spectrum antibiotics or combination of antibiotics has to be started empirically after obtaining appropriate cultures. ${ }^{26}$ As susceptibility rates to cephalosporins are severely decreasing, cephalosporins should not be a preferable choice for empirical therapy. ${ }^{3}$

Carbapenems are the treatment of choice for empirical therapy of severe infections caused by ESBL-producing organisms. ${ }^{40}$

\section{Current Treatment Options for Treating MDR GNB Infections}

- Carbapenems

- Polymyxins

- Fosfomycin

- Plazomicin

- Tigecycline

- Eravacycline.

Betalactam-betalactamase inhibitors (BL-BLIs): Ceftazidime/ avibactam, meropenem/vaborbactam, and ceftolozane/ tazobactam. 


\section{Carbapenems (Meropenem and Imepenem)}

Carbapenems are the drug of choice for treating life-threatening infections caused by ESBL-producing GNB. Studies have shown that 2-week mortality in patients with Klebsiella pneumoniae. BSI treated with meropenem was very less compared to other betalactam antibiotics. ${ }^{41}$ Carbapenems have wide distribution in different sites of the body like lung, blood, urine, and cerebrospinal fluid. ${ }^{42}$ They act in time-dependent bacterial fashion; $40-50 \%$ of the time, the drug levels have to be above the MIC. ${ }^{42}$ As they are excreted unchanged through kidney, to maintain their concentration constantly above MIC, they can be administered as continuous infusion. ${ }^{43}$

For treating susceptible KPC-producing Klebsiella infection, colistin and meropenem combination can be used when the MIC is $8 \mathrm{mg} / \mathrm{L}$ and prolonged meropenem infusion should be considered if the MIC is $>8$ but $<32 \mathrm{mg} / \mathrm{L}^{.44}$

\section{Polymyxins}

Polymyxins are bactericidal agents which act on outer membrane of GNB.

They bind with the anionic lipopolysaccharide molecules by displacing calcium and magnesium from the outer cell membrane, leading to permeability changes in the cell envelope, leading to leakage of cell contents and cell death. ${ }^{45}$

Colistin (polymyxin E) and polymyxin B are in wide usage, and colistin is parentrally available in the form of colistimethate sodium (CMS) which gets hydrolyzed to colistin and sulfomethylated derivatives once it enters the body. It acts in a concentrationdependent fashion and is excreted through kidney. ${ }^{45}$

\section{Colistin-loading Dose}

As discussed earlier, the parentral CMS has to get converted to active colistin which is slow process, and colistin kills the bacteria in a concentration-dependent fashion. In view of large volume of distribution in critically ill patients and as the half-life of colistin is 2-3 days, it is important to give colistin-loading dose to achieve higher concentrations rapidly. ${ }^{46,47}$ The loading doses do not affect kidney function, but the maintenance doses have to be adjusted depending on the kidney function. ${ }^{48}$

Colistin nebulization should be used in addition to intravenous therapy in patients with VAP due to MDR GNB. ${ }^{49}$ Colistin combination therapy: colistin with meropenem combination had better outcome than any other combination with colistin. MIC for colistin and meropenem combination is $0.12 \mu \mathrm{g} / \mathrm{mL}$, which is fivefold less when compared to meropenem $4 \mu \mathrm{g} / \mathrm{mL}$ and colistin $0.38 \mu \mathrm{g} / \mathrm{mL}$ when tested as single agents. ${ }^{50}$ Colistin can be combined with amikacin or tigecycline in treating carbapenem-resistant GNB infections if they are sensitive to these antibiotics. ${ }^{44}$

\section{Fosfomycin}

Similar to colistin fosfomycin is also an old broad-spectrum bactericidal antibiotic, which acts on bacterial cell wall by interfering with formation of UDP N-acetylmuramic acid, a peptidoglycan precursor. It is used in treating MDR GNB infections often in combination with other agents when there are no alternatives. ${ }^{51}$

\section{Tigecycline}

It is a newer agent in tetracycline class which has shown good activity against CRE. Pseudomonas aeruginosa is inherently resistant to tigecycline. ${ }^{52}$ Tigecycline rapidly redistributes into tissues and achieves low serum levels; hence, it is not useful in treating BSIs and VAP. $^{53}$
Food and Drug Administration (FDA) has approved tigecycline only in the treatment of soft tissue infection and IAls caused by MDR GNB. ${ }^{44}$

\section{Eravacycline}

It is newer drug structurally similar to tigecycline, and it has been approved by FDA and European Medicines Agency (EMA) approved for the treatment of complicated IAIs (cIAIs). It is not affected by efflux pumps and ribosomal alterations by the GNBs, which is unique when compared to other tetracyclines. It has in vitro activity against KPCproducing bacteria and also Acenatobacter baumannii. It has $90 \%$ oral bioavailability and also available in intravenous preparation. It has broad-spectrum of action covering gram-positive, GNB, and anaerobic bacteria except Pseudomonas and burkholdaria. ${ }^{54}$

\section{Plazomicin}

It is a newer aminoglycoside, and it stable against several aminoglycoside-modifying enzymes. It is active against CRE.

It is currently approved by the FDA for the treatment of complicated UTI (cUTI). ${ }^{55}$

\section{Ceftazidime/Avibactam}

This combination has been approved by FDA and EMA for cUTIs, clAls, hospital-acquired pneumonia (HAP), and VAP. Studies showed a lower 30-day mortality with this combination for treating bacteriemia caused by KPC-producing Klebsiella isolates. ${ }^{56}$

Hence, ceftazidime/avibactam is an effective option for the treatment of CRE. This combination is available in India.

\section{Meropenem/Vaborbactam}

It is another new BL-BLI, and it has a potent activity against class A (e.g., KPC) carbapenemase-producing CRE. It is FDA and EMA approved for CUTI, CIAI, HAP, and VAP in adult patients with limited treatment options. ${ }^{57}$

Ceftolozane/tazobactam: it is another newer BL-BLI, and it is effective against $P$ seudomonas infections, but it is not active against CRE. It is approved by FDA and EMA for the treatment of CIAI and CUTI. ${ }^{58,59}$

\section{Proposed Treatment Options for MDR-GNB Infections in Critically III Patients ${ }^{57}$}

\section{Current Treatment Options for CRE}

- Ceftazidime/avabactam (as preferred empirical choice when both KPC and OXA carbapenemases are reported locally) or meropenem/vabrobactam.

- For empirical therapy-add aminoglycoside or polymyxin or fosfomycin.

- In case of resistance to newer BL-BLI: consider polymyxin or aminoglycoside based combinations with carbapenems and/ or fosfomycin and/or rifampin.

- For empirical and targeted therapy: combination with old (colistin, polymyxin B, old aminoglycosides, and fosfomycin) or novel agents (plazomicin, eravacycline, and double BL-BLI) could be considered on case-to-case basis (although lack of high-level evidence).

- Concomitant use inhaled polymyxins/aminoglycosides when they are used intravenously for VAP.

\section{Current Treatment Options for Carbapenem Resistance Pseudomonas arugenosa}

- Ceftolazone/tazobactam (as preferred empirical choice in absence of concomitant risk of CRE) or ceftazidime/avabactam. 
- For empirical therapy, administer a second antipseudomonal agent (an aminoglycoside or polymyxin or fosfomycin).

- In case of resistance to newer BL-BLI: consider polymyxin or aminoglycoside based combinations with carbapenems and/ or fosfomycin and/or rifampin.

- For targeted therapy: combination with old (colistin, polymyxin $B$, old aminoglycosides, and fosfomycin) or novel agents (plazomicin and double BLBLI) could be considered on case-tocase basis (although lack of high-level evidence).

- Concomitant use inhaled polymyxins/aminoglycosides when they are used intravenously for VAP.

\section{Current Treatment Options for Carbapenem Resistance Acinetobacter baumannii}

- Administer polymyxin as the backbone agent.

- Consider combination with old (carbapenems, aminoglycosides, tigecycline, fosfomycin, and rifampin) or novel agents (plazomicin and eravacycline).

- Concomitant use inhaled polymyxins/aminoglycosides when they are used intravenously for VAP.

\section{Key Points}

- Incidence of carbapenemase-producing GNB infections are rapidly growing in Indian ICUs, especially NDM-1-producing gram negatives.

- Assessment of risk factors, MIC testing, and proper interpretation of antibiogram are vital in identifying these ESBL carbapenemaseproducing and MDR GNB.

- Carbapenems are the drug of choice in treating serious infections caused by ESBL producers and are advised at high doses for carbapenemase-producing organisms (Klebsiella spp.) in combination with colistin as empirical therapy.

- Colistin is the backbone agent in treating carbapenem-resistant Acinetobacter baumannii, and loading dose has to be given to reach adequate drug concentration in critically ill patients.

- Inhaled colistin is advised in treating VAP along with intravenous colistin.

- Newer agents like plazomicin, eravacycline, fosfomycin, and BL-BLI combinations can be combined with colistin and meropenem for treating carbapenemase producers.

\section{ConcLusion}

- All hospitals should have their own institutional antimicrobial stewardship program, and it should be based on local antibiograms and international guidelines to prevent the emergence of MDR GNB and even a more complex situation.

- Infection control practices should be strictly implemented to prevent MDR GNB infections in ICUs.

- Differentiate true infection from colonization and avoid unnecessary antibiotic prescriptions.

- Suspect MDR GNB infections and start adequate empirical antibiotics as soon as possible as delay in starting appropriate antibiotic is associated with poor outcomes.

- Deescalate to appropriate antibiotic as soon as the sensitivity pattern is available.

\section{References}

1. Boucher HW, Talbot GH, Bradley JS, et al. Bad bugs, no drugs: no ESKAPE! An update from the Infectious Diseases Society of America. Clin Infect Dis 2009;48(1):1-12. DOI: 10.1086/595011.
2. Vasoo S, Barreto JN, Tosh PK. Emerging issues in Gram-negative bacterial resistance: an update for the practicing clinician. Mayo Clin Proc 2015;90(3):395-403. DOI: 10.1016/j.mayocp.2014.12.002.

3. Veeraraghavan B, Jesudason M, Prakasah J, et al. Antimicrobial susceptibility profiles of gram-negative bacteria causing infections collected across India during 2014-2016: Study for monitoring antimicrobial resistance trend report. Indian J Med Microbiol 2018;36(1):32-36. DOI: 10.4103/ijmm.IJMM_17_415.

4. Kumar SG, Adithan C, Harish BN, et al. Antimicrobial resistance in India: a review. J Nat Sci Biol Med 2013;4(2):286-291. DOI: 10.4103/09769668.116970

5. Centers for Disease Control and Prevention (CDC). Vital signs: carbapenem-resistant Enterobacteriaceae. MMWR Morb Mortal Wkly Rep 2013;62(9):165-170.

6. Rossi F. In vitro susceptibilities of aerobic and facultatively anaerobic Gram-negative bacilli isolated from patients with intra-abdominal infections worldwide: 2004 results from SMART (Study for Monitoring Antimicrobial Resistance Trends). J Antimicrob Chemother 2006;58(1):205-210. DOI: 10.1093/jac/dkl199.

7. Hsueh PR. Study for monitoring antimicrobial resistance trends (SMART) in the Asia-Pacific region, 2002-2010. Int J Antimicrob Agents 2012;40(Suppl):S1-S3. DOI: 10.1016/S0924-8579(12)00244-0.

8. Doi Y, Adams J, O'Keefe A, et al. Community-acquired extendedspectrum beta-lactamase producers, United States. Emerg Infect Dis 2007;13(7):1121-1123. DOI: 10.3201/eid1307.070094.

9. Freeman JT, McBride SJ, Heffernan $\mathrm{H}$, et al. Community-onset genitourinary tract infection due to CTX-M-15-Producing Escherichia coli among travelers to the Indian subcontinent in New Zealand. Clin Infect Dis 2008;47(5):689-692. DOI: 10.1086/590941.

10. Woodford N, Ward ME, Kaufmann ME, et al. Community and hospital spread of Escherichia coli producing CTX-M extended-spectrum beta-lactamases in the UK. J Antimicrob Chemother 2004;54(4): 735-743. DOI: 10.1093/jac/dkh424.

11. Hawser SP, Bouchillon SK, Hoban DJ, et al. Emergence of high levels of extended spectrum beta lactamase producing gram negative Bacilli in the Asia Pacific region: data from the study for monitoring antimicrobial resistance trends (SMART) program, 2007. Antimicrob Agents Chemother 2009;53(8):3280-3284. DOI: 10.1128/AAC. 00426-09.

12. Chaudhry D, Prajapat B. Intensive care unit bugs in India: how do they differ from the Western world? J Assoc Chest Physicians 2017;5:10-17. DOI: $10.4103 / 2320-8775.196645$.

13. Rodriguez-Baño J, Pascual A. Clinical significance of extendedspectrum beta-lactamases. Expert Rev Anti Infect Ther 2008;6(5): 671-683. DOI: 10.1586/14787210.6.5.671.

14. Gasink L, Edelstein P, Lautenbach E, et al. Risk factors and clinical impact of Klebsiella pneumoniae carbapenemase-producing $\mathrm{K}$. pneumoniae. Infect Control Hosp Epidemiol 2009;30(12):1180-1185. DOI: $10.1086 / 648451$.

15. Mouloudi E, Protonotariou E, Zagorianou A, et al. Bloodstream infections caused by metallo- $\beta$-lactamase/Klebsiella pneumoniae carbapenemase-producing K. pneumoniae among intensive care unit patients in Greece: risk factors for infection and impact of type of resistance on outcomes. Infect Control Hosp Epidemiol 2010;31(12):1250-1256. DOI: 10.1086/657135.

16. Magiorakos A, Srinivasan A, Carey R, et al. Multidrug-resistant, extensively drug-resistant and pandrug-resistant bacteria: an international expert proposal for interim standard definitions for acquired resistance. Clin Microbiol Infect 2012;18(3):268-281. DOI: 10.1111/j.1469-0691.2011.03570.x.

17. Jacoby GA, Munoz-Price LS. The new beta-lactamases. N Engl J Med 2005;352(4):380-391. DOI: 10.1056/NEJMra041359.

18. Ambler R. The structure of beta-lactamases. Philos Trans R Soc Lond B Biol Sci 1980;289(1036):321-331. DOI: 10.1098/rstb.1980.0049.

19. Bush K, Jacoby G. Updated functional classification of betalactamases. Antimicrob Agents Chemother 2010;54(3):969-976. DOI: 10.1128/AAC.01009-09.

20. Canton R, Coque T. The CTX-M betalactamase pandemic. Curr Opin Microbiol 2006;9(5):466-475. DOI: 10.1016/j.mib.2006.08.011. 
21. Tzouvelekis L, Markogiannakis A, Psichogiou M, et al. Carbapenemases in Klebsiella pneumoniae and other Enterobacteriaceae:an evolving crisis of global dimensions. Clin Microbiol Rev 2012;25(4):682-707. DOI: 10.1128/CMR.05035-11.

22. Jacoby G. AmpC beta-lactamases. Clin Microbiol Rev 2009;22(1): 161-182. DOI: 10.1128/CMR.00036-08.

23. Navarro F, Miró E, Mirelis $B$. Interpretive reading of enterobacteriaantibiograms. Enferm Infecc Microbiol Clin 2010;28(9):638-645. DOI: 10.1016/j.eimc.2010.05.002.

24. Poirel L, Heritier C, Tolun V, et al. Emergence of oxacillinase-mediated resistance to imipenem in Klebsiella pneumoniae. Antimicrob Agents Chemother 2004;48(1):15-22. DOI: 10.1128/AAC.48.1.15-22.2004.

25. Delgado-Valverde M, Sojo-Dorado J, Pascual A, et al. Clinical management of infections caused by multidrug-resistant Enterobacteriaceae. Ther Adv Infect Dis 2013;1(2):49-69. DOI: $10.1177 / 2049936113476284$.

26. Pop-Vicas A, Opal SM. The clinical impact of multidrugresistant gram-negative bacilli in the management of septic shock. Virulence 2014;5(1):206-212. DOI: 10.4161/viru.26210.

27. Cornaglia G, Giamarellou H, Rossolini GM. Metallo $\beta$-lactamases: a last frontier for $\beta$-lactams? Lancet Infect Dis 2011;11(5):381-393. DOI: 10.1016/S1473-3099(11)70056-1.

28. Logan LK. Carbapenem-resistant enterobacteriaceae: an emerging problem in children. Clin Infect Dis 2012;55(6):852-859. DOI: 10.1093/ $\mathrm{cid} / \mathrm{cis} 543$.

29. Miriagou V, Tzouvelekis LS, Rossiter $S$, et al. Imipenem resistance in a Salmonella clinical strain due to plasmid-mediated class A carbapenemase KPC-2. Antimicrob Agents Chemother 2003;47(4):1297-1300. DOI: 10.1128/AAC.47.4.1297-1300.2003.

30. Castanheira M, Deshpande LM, Mathai D, et al. Early dissemination of NDM-1- and OXA-181-producing Enterobacteriaceae in Indian hospitals: report from the SENTRY antimicrobial surveillance program, 2006-2007. Antimicrob Agents Chemother 2011;55(3): 1274-1278. DOI: 10.1128/AAC.01497-10.

31. Pragasam AK, Vijayakumar S, Bakthavatchalam YD, et al. Molecular characterisation of antimicrobial resistance in Pseudomonas aeruginosa and Acinetobacter baumannii during 2014 and 2015 collected across India. Indian J Med Microbiol 2016;34(4):433-441. DOI: 10.4103/0255-0857.195376.

32. Piddock LJ. Mechanisms of fluoroquinolone resistance: an update 1994-1998. Drugs 1999;58(Suppl 2):11-18. DOI: 10.2165/00003495$199958002-00003$.

33. Doi Y, Arakawa Y. 16 S ribosomal RNA methylation: emerging resistance mechanism against aminoglycosides. Clin Infect Dis 2007;45(1):88-94. DOI: 10.1086/518605.

34. Zhou Y, Yu H, Guo Q, et al. Distribution of $16 \mathrm{~S}$ rRNA methylases among different species of Gram-negative bacilli with high-level resistance to aminoglycosides. Eur J Clin Microbiol Infect Dis 2010;29(11): 1349-1353. DOI: 10.1007/s10096-010-1004-1.

35. Bonomo RA, Szabo D. Mechanisms of multidrug resistance in Acinetobacter species and Pseudomonas aeruginosa. Clin Infect Dis 2006;43(Suppl 2):S49-S56. DOI: 10.1086/504477.

36. Vincent JL, Rello J, Marshall J, et al. International study of the prevalence and outcomes of infection in intensive care units. JAMA 2009;302(21):2323-2329. DOI: 10.1001/jama.2009.1754.

37. Kumar A, Roberts D, Wood KE, et al. Duration of hypotension before initiation of effective antimicrobial therapy is the critical determinant of survival in human septic shock. Crit Care Med 2006;34(6): 1589-1596. DOI: 10.1097/01.CCM.0000217961.75225.E9.

38. Tumbarello M, Sanguinetti M, Montuori E, et al. Predictors of mortality in patients with bloodstream infections caused by extended-spectrum-beta-lactamase-producing Enterobacteriaceae: importance of inadequate initial antimicrobial treatment. Antimicrob Agents Chemother 2007;51(6):1987-1994. DOI: 10.1128/AAC.01509-06.

39. Leekha S, Standiford HC. Empiric antimicrobial therapy for Gramnegative sepsis: back to the future. Crit Care Med 2011;39(8): 1995-1996. DOI: 10.1097/CCM.0b013e318223b94b.

40. Hawkey PM, Livermore DM. Carbapenem antibiotics for serious infections. BMJ 2012;344:e3236. DOI: 10.1136/bmj.e3236.
41. Paterson DL, Ko WC, Von Gottberg A, et al. Antibiotic therapy for Klebsiella pneumoniae bacteremia: implications of production of extended-spectrum beta-lactamases. Clin Infect Dis 2004;39(1):31-37. DOI: $10.1086 / 420816$.

42. Drusano GL. Antimicrobial pharmacodynamics: critical interactions of 'bug and drug'. Nat Rev Microbiol 2004;2(4):289-300. DOI: 10.1038/ nrmicro862.

43. Roberts JA, Kirkpatrick CM, Roberts MS, et al. Meropenem dosing in critically ill patients with sepsis and without renal dysfunction: intermittent bolus versus continuous administration? Monte Carlo dosing simulations and subcutaneous tissue distribution. J Antimicrob Chemother 2009;64(1):142-150. DOI: 10.1093/jac/dkp139.

44. Hawkey P, Warren R, Livermore D, et al. Treatment of infections caused by multidrug-resistant Gram-negative bacteria: report of the British Society for Antimicrobial Chemotherapy/Healthcare Infection Society/British Infection Association Joint Working Party. J Antimicrob Chemother 2018;73(Suppl 3):iii2-iii78. DOI: 10.1093/jac/dky027.

45. Falagas M, Kasiakou S, Saravolatz L. Colistin: the revival of polymyxins for the management of multidrug-resistant gram-negative bacterial infections. Clin Infect Dis 2005;40(9):1333-1341. DOI: 10.1086/ 429323.

46. Kift EV, Maartens G, Bamford C. Systematic review of the evidence for rational dosing of colistin. S Afr Med J 2014;104(3):183-186. DOI: 10.7196/samj.7011.

47. Landersdorfer CB, Nation RL. Colistin: how should it be dosed for the critically ill? Crit Care Med 2015;36(1):126-135. DOI: 10.1055/s-00341398390.

48. Labuschagne Q, Schellack N, Gous A, et al. COLISTIN: adult and paediatric guideline for South Africa, 2016. S Afr J Infect Dis 2016;31(1):3-7. DOI: 10.1080/23120053.2016.1144285.

49. Michalpulos A, Papadakis E. Inhaled anti-infective agents: emphasis on colistin. Infection 2010;38(2):81-88. DOI: 10.1007/s15010-009-9148-6.

50. Balaji V, Jeremiah SS, Baliga PR. Polymyxins: Antimicrobial susceptibility concerns and therapeutic options. Indian J Med Microbiol 2011;29(3):230-242. DOI: 10.4103/0255-0857.83905.

51. Falagas ME, Kastoris AC, Kapaskelis AM, et al. Fosfomycin for the treatment of multidrug-resistant, including extended-spectrum beta-lactamase producing, Enterobacteriaceae infections: a systematic review. Lancet Infect Dis 2010;10(1):43-50. DOI: 10.1016/ S1473-3099(09)70325-1.

52. Pankey GA. Tigecycline. J Antimicrob Chemother 2005;56(3):470-480. DOI: $10.1093 / \mathrm{jac} / \mathrm{dki} 248$

53. Anthony KB, Fishman NO, Linkin DR, et al. Clinical and microbiological outcomes of serious infections with multidrug-resistant gramnegative organisms treated with tigecycline. Clin Infect Dis 2008;46(4):567-570. DOI: 10.1086/526775.

54. Bassetti M, Righi E, Carnelutti A. New therapeutic options for respiratory tract infections. Curr Opin Infect Dis 2016;29(2):178-186. DOI: $10.1097 /$ QCO.0000000000000251.

55. Wagenlehner FME, Cloutier DJ, Miller LG, et al. Once-daily plazomicin for complicated urinary tract infections. N Engl J Med 2019;380(8):729-740. DOI: 10.1056/NEJMoa1801467.

56. Tumbarello M, Trecarichi EM, Corona A, et al. Efficacy of ceftazidimeavibactam salvage therapy in patients with infections caused by KPCproducing Klebsiella pneumoniae. Clin Infect Dis 2019;68(3):355-364. DOI: 10.1093/cid/ciy492.

57. Bassetti $M$, Peghin $M$, Vena $A$, et al. Treatment of infections due to MDR Gram-negative bacteria. Front Med (Lausanne) 2019;6:74. DOI: 10.3389/fmed.2019.00074.

58. Solomkin J, Hershberger E, Miller B, et al. Ceftolozane/tazobactam plus metronidazole for complicated intraabdominal infections in an era of multidrug resistance: results from a randomized, double-blind, phase 3 trial. (ASPECT-CIAI). Clin Infect Dis 2015;60(10):1462-1471. DOI: 10.1093/cid/civ097.

59. Wagenlehner FM, Umeh O, Steenbergen J, et al. Ceftolozanetazobactam compared with levofloxacin in the treatment of complicated urinary-tract infections, including pyelonephritis: a randomised, double-blind, phase 3 trial (ASPECT-CUTI). Lancet 2015;385(9981):1949-1956. DOI: 10.1016/S0140-6736(14)62220-0. 